Arab Journal of Nuclear Sciences and Applications

Web site: ajnsa.journals.ekb.eg

\title{
A Study on the Effect of Cathode Electrode Aperture Shapes on Ion Beam Properties Using SIMION Program
}

\author{
B.A.Soliman* and M.M. Abdelrahman \\ Accelerators \& Ion Sources Department, Nuclear Research Centre, Atomic Energy Authority, Cairo, Egypt
}

\begin{tabular}{l} 
ARTICLE INFO \\
\hline Article history: \\
Received: $8^{\text {th }}$ June 2020 \\
Accepted: $8^{\text {th }}$ Mar. 2021 \\
\hline Keywords: \\
Ion Beam Simulation, \\
SIMION Program, \\
Beam Optics, \\
Cathode Electrode Aperture.
\end{tabular}

\begin{abstract}
This work involves a simulation study of different cathode electrode aperture shapes (pierce, reversed pierce, cone, reversed cone, triangle, reversed triangle, rectangle, circle, and sphere), which confirms that pierce and cone shapes are the best extraction apertures. The minimum values of ion beam diameter and ion beam emittance, at collector plate placed at $30 \mathrm{~mm}$ distance from cathode electrode, were obtained using pierce and cone shapes. Moreover, the distance of the ion beam trajectories focal point was studied using different shapes of cathode aperture, which was found to decrease with increasing the voltage applied to the cathode electrode. The main parameter in this study was the applied voltage to the cathode electrode. It was found that the observed changes on the ion beam parameters are in the range $100-1500 \mathrm{~V}$, but at charges higher than these values the behavior of diameter, emittance and focal point of the ion beam were quasi-saturated. It was also found that the general behavior of ion beam diameter and its emittance nearly the same.
\end{abstract}

\section{INTRODUCTION}

Simulation programs for ion beam extraction and transport are very useful tools before experimental setup, that help the designer in selecting the electrodes shape and the values of electric or magnetic fields for many applications [1 - 3]. Ion beam extraction is the intermediate stage between plasma formation and beam transport for different applications. Different types of extraction systems can be used to extract the ions from the plasma [4 - 10]. The type of the extraction system depends on the ion source type and the plasma formation method. The most common shapes of the extraction systems are considering pierce electrode with an angle $67.5^{\circ}$ with ion beam trajectories. The extraction system is playing a main role in beam emittance, diameter and the distance of the focal point.

In the present work, the extraction of ions will be diffused from the source through cathode electrode, under the assumption that of ion beams self-extraction. The plasma density, energy, and the angular distributions of the extracted ions depend on the shape of the plasma meniscus formed over the cathode electrode. The extraction of ions from the plasma region depends upon the interaction of the plasma with the surface of the cathode electrode. The geometry of the cathode electrode plays a vital role in the efficient extraction of the ions and the angular divergence of the ion beam.

The aim of this work is to control the ion beam width, where it can be determined by shape of cathode electrode. Ion beam quality is determined by many parameters (emmitance, brightness, diameter, perveance... etc.), which are affecting ion beam trajectory (divergent, convergent, parallel or focus). Consequently, according to different ion beam applications, these parameters are optimized. Ion beam diameter and its emittance were controlled by many parameters such as; acceleration voltage, extraction gap width, acceleration system, focusing system. In this study, only the extraction electrode was considered, specially, its aperture shape to focus or defocus the ion beam. This can be done by mechanical change during electrode manufacture without need electric or magnetic fields. There are many studies on the shape of extraction electrode, but there are few specific studies on the shape of the extraction electrode aperture [11, 12], (in the 
present work the expression of cathode electrode aperture or plasma electrode aperture refers to the extraction electrode aperture). When the shape of an extraction aperture is changed, it will have effects on surrounding electric field, and the trace length of ion beam in the aperture.

\section{Simulation of ion beam trajectories}

Simulation programs are useful tools in simulating trajectories of ion beam and predicting the track of ions. Other parameters of ion beam can also be deduced such as; beam emittance, beam diameter ...etc. which determine the behavior along its path (i.e. convergent, divergent, focused or parallel). One of these programs is SIMION 3D 7.0; which is a full windows program thatcan be used in drawing the electrical or magnetic lenses along beam track. The original version has been developed by D.C.McGilvery at Latrobe University in Australia (1977) [13]. Other ion beam optics are; beam transport, accel / decel system, analyzing charged particles lenses, ion traps, accelerator system ...etc. On the other hand, the ion beam is influenced by many parameters [14] such as the plasma boundary shape, cathode/plasma electrode shape, space charge and extraction voltage. The parameters of ion beam used in SIMION program were as follows:

- Plasma boundary shape is flat.

- Cathode/plasma electrode was of disk shape with different aperture shapes.

- The extraction voltage is in the range $0-5000 \mathrm{~V}$ (Cathode/plasma electrode voltage $\mathrm{V}_{\mathrm{p}}$ ).

- Without space charge.

- The ion beam outlet aperture is $2 \mathrm{~mm}$.

- The distance between cathode/plasma electrode and collector plate is $30 \mathrm{~mm}$.

\section{RESULTS AND DISCUSSIONS}

Fig. (1) shows the different cathode aperture shapes which are drawn up by SIMION program. These aperture shapes have an observed effect on the beam parameters, where the plasma boundary was considered as flat, and the collector plate placed at $30 \mathrm{~mm}$ distance from cathode/plasma electrode. Fig.(2) shows the arrangement of electrodes in the simulation program with electric field distribution and ions flay, the aperture is circular in shape.

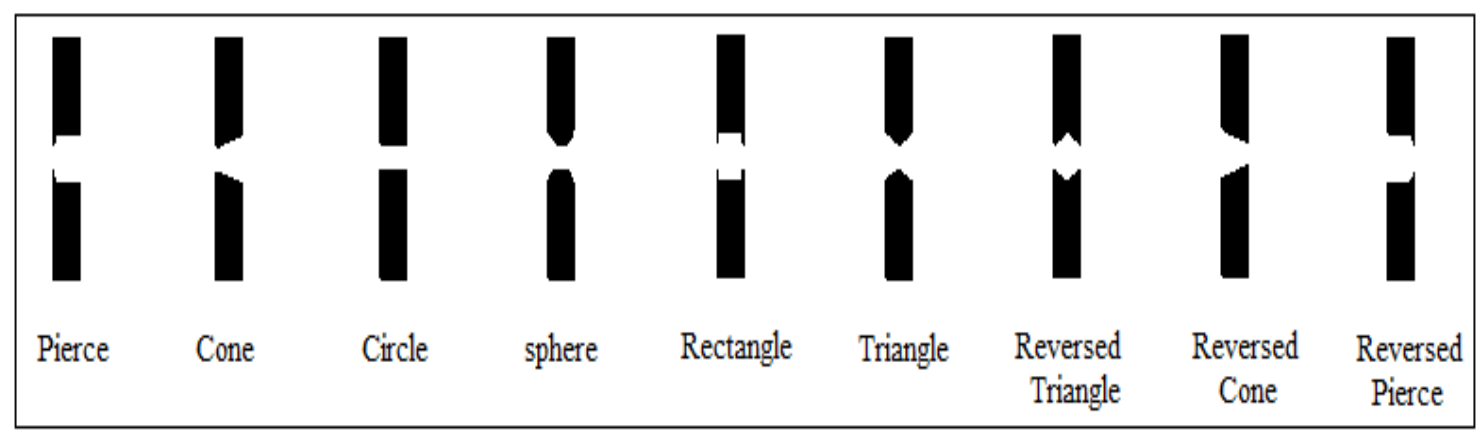

Fig. (1): Different shapes of outlet cathode/plasma electrode aperture

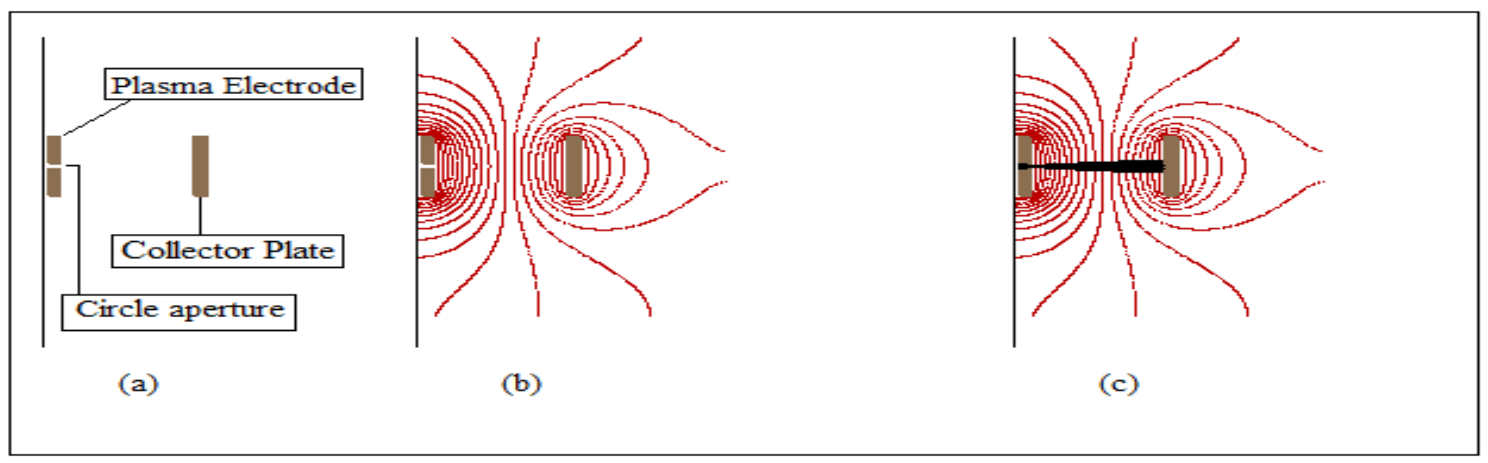

Fig. (2): An example for simulation process; (a) Electrodes, (b) Electric field distribution between electrodes and (c) Ions flay 
Fig.(3) shows the behavior of the ion beam emittance with increasing $V_{p}$, where it can be observed that pierce, cone, rectangle and reversed triangle shapes gave the lowest values of ion beam emittance, but the shape of reversed pierce gave the highest values. While the other aperture shapes gave the values in between. Also, the reversed pierce's curve gave a maximum value at $\mathrm{V}_{\mathrm{p}}=700 \mathrm{~V}$. Fig. (4) shows the difference in ion beam emittance as a function in ion beam diameter, for pierce and reversed pierce apertures, at the same $\mathrm{V}_{\mathrm{p}}=700 \mathrm{~V}$. The parameters in the two shapes ( $a$ and $b$ ) were the same except for the aperture shape of cathode/plasma electrode. These shapes have an effect on plasma meniscus, the distribution of electric field lines and ion beam particles trajectory [8].

Fig.(5) shows the behavior of the ion beam diameter with increasing $V_{p}$, where it can be observed that the pierce and cone shapes were giving the lowest values of ion beam diameter $\approx 4 \mathrm{~mm}$, but its highest values were given by the shape of reversed pierce $\approx 13$ $\mathrm{mm}$ and reversed cone $\approx 10 \mathrm{~mm}$, (approximately 3 times wider, see Fig. 4) while other aperture shapes gave the values in between, and the same behavior of ion beam emittance.

Fig.(6) shows the behavior of the focal point with increasing $\mathrm{V}_{\mathrm{p}}$. It is clear that all the shapes have the same behavior and their values were approximately equal, where the shape of aperture has no effect on the focal point behavior. Additionally, there are negative values of the focal point position which means that the focal point was inside the aperture of cathode/plasma electrode. This has also occurred with most of shapes except the rectangle, reversed triangle, and reversed pierce shapes which have Isharp edges leading to a strong electric field at theseedges.

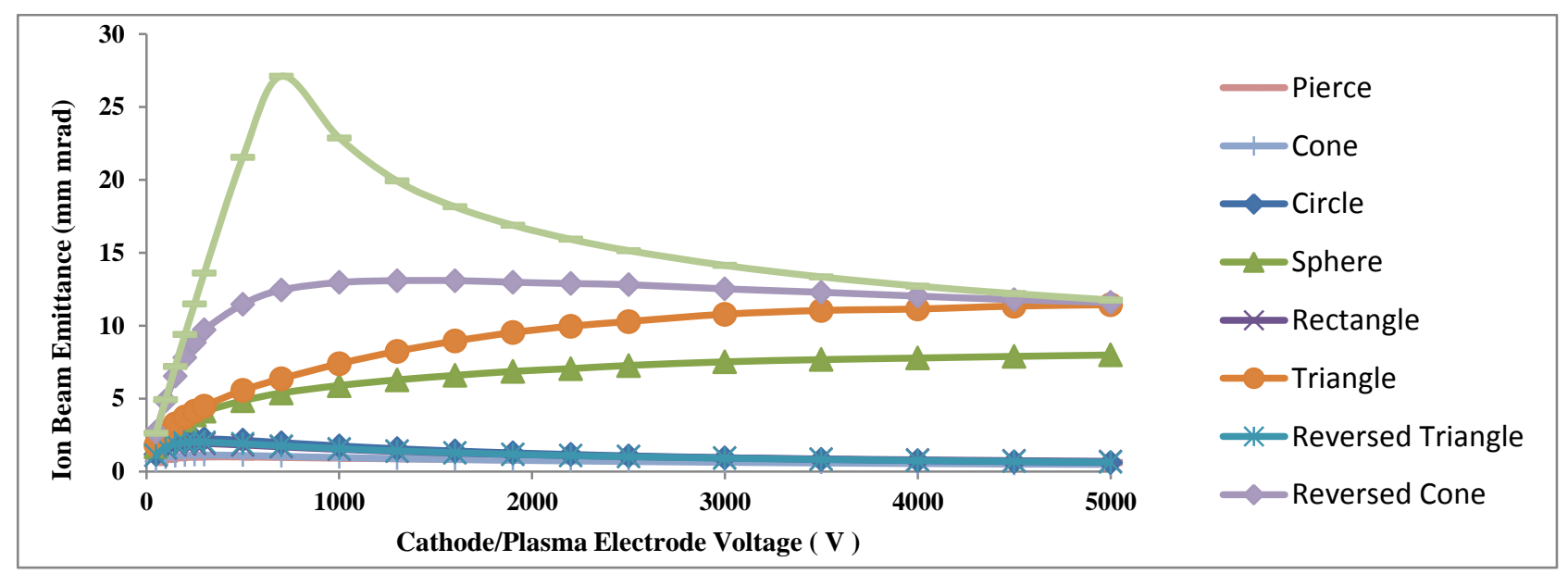

Fig. (3): The relation between cathode/plasma electrode voltage and ion beam emittance

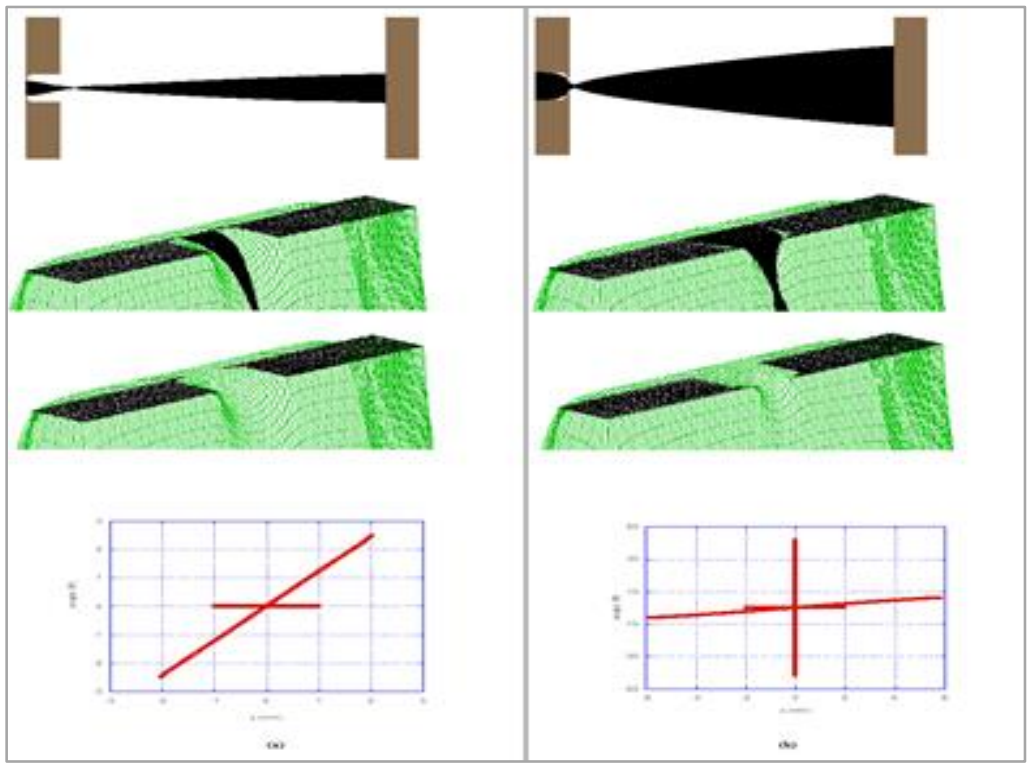

Fig. (4): Effect of aperture shape of cathode/plasma electrode on ion beam emittance and diameter, at $\mathrm{Vp}=1000 \mathrm{~V}$ (a) pierce (b) reversed pierce 


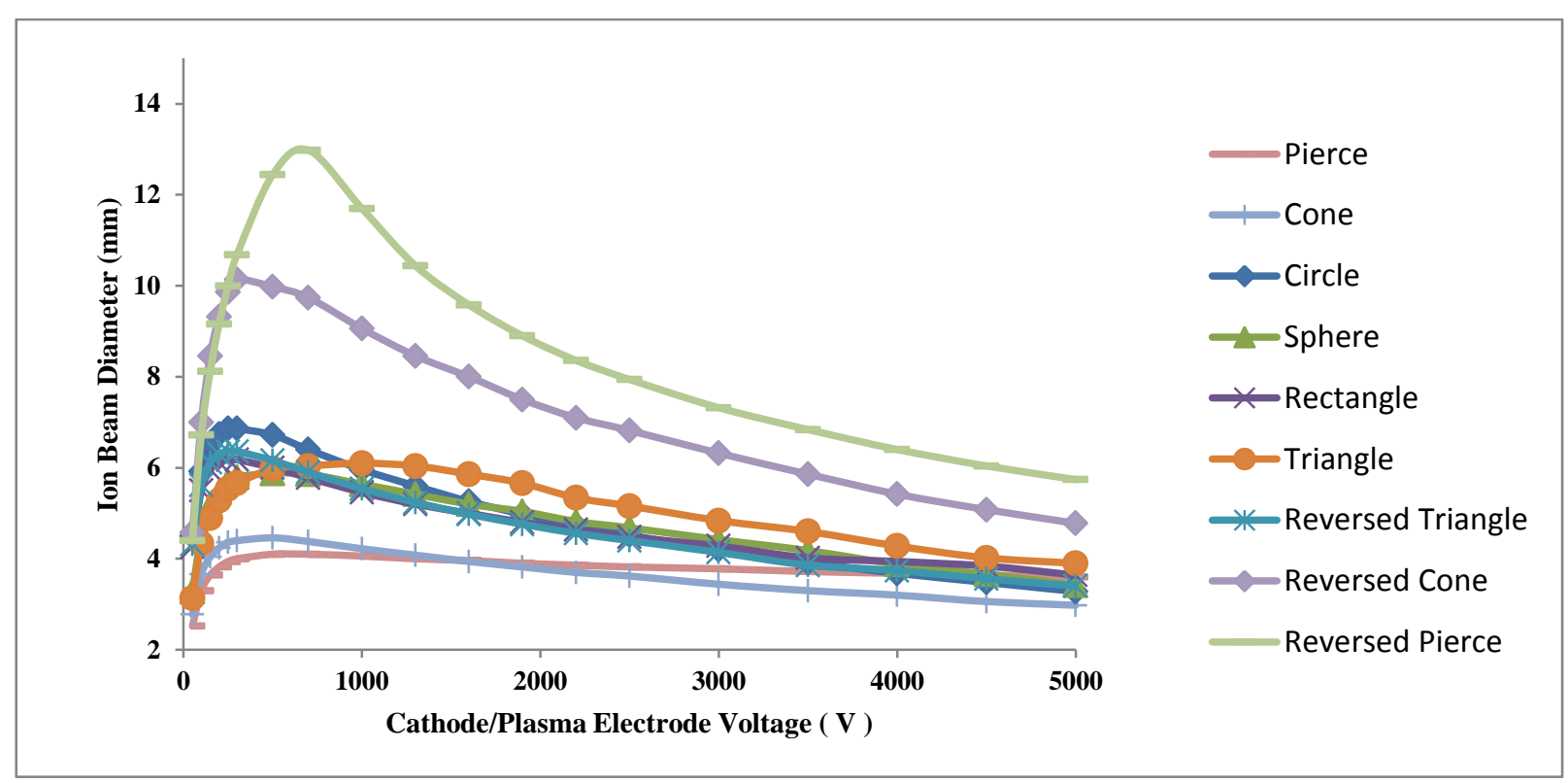

Fig. (5): The relation between cathode/plasma electrode voltage and ion beam diameter

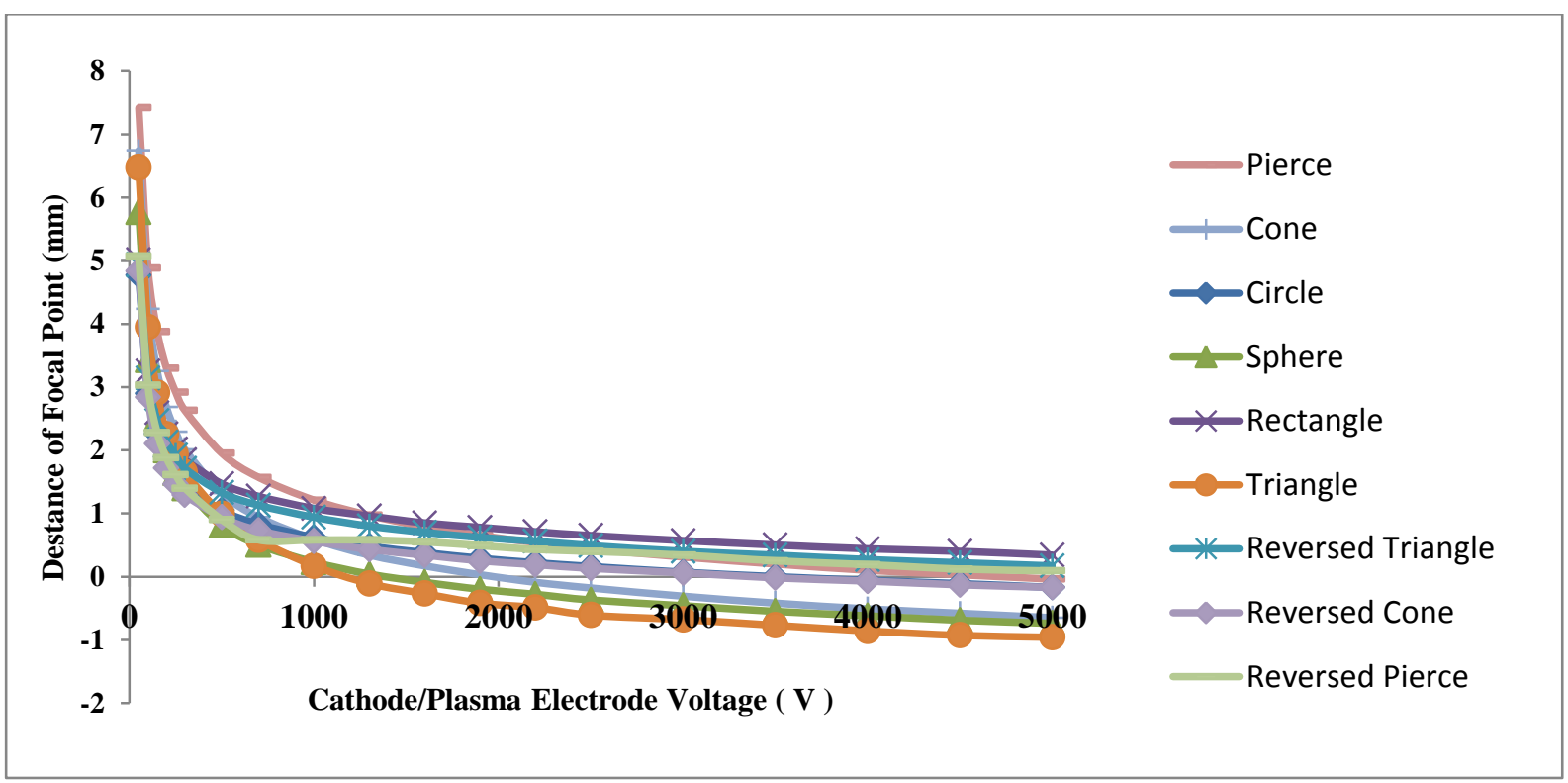

Fig. (6): The relation between cathode/plasma electrode voltage and ion beam focal point

\section{CONCLUSION}

The results of the present study were very useful in the experimental work to irradiate the samples using suitable ion beam diameters. From the simulation results, it was found that the ion beam diameter can be multiplied many times by invers plasma electrode surface to opposite surface to use for different size samples irradiation. Moreover, it was concluded that the irradiation of different size samples can be performed in the range from $2 \mathrm{~mm}$ up to $13 \mathrm{~mm}$ at plasma electrode voltage in the range from $100 \mathrm{~V}$ up to $1500 \mathrm{~V}$.

\section{REFERENCES}

[1] H. Mikage, M. Kitagawa, and M. Wada (2020) Development of a magnetic quadrupole lens for low energy heavy ion beam transport,Rev. Sci. Instrum. 91, 015107.

[2] Yosuke Yuri, Mitsuhiro Fukuda and Takahiro Yuyama (2019) 10th International Particle Accelerator Conference, Journal of Physics: Conference Series 1350, 012115, IOP Publishing.

[3] A. Kurup et al (2019) Simulation of a radiobiology facility for the Centre for the Clinical Application 
of Particles, European Journal of Medical Physics, Vol. 65, 21-28.

[4] P. Spädtke, (2018) Invited Review Article: Modeling ion beam extraction from different types of ion sources, Rev. Sci. Instrum. 89, 081101.

[5] M.M. Abdelrahman and S.G. Zakhary, (2009) Simulation Studies for Ion Beam Extraction Systems, Brazilian Journal of Physics, vol. 39, no. 2, 275-279.

[6] Sae-Hoon Park and Yu-Seok Kim, (2017) Simulation Study on Duoplasmatron with Optimization of Ion Beam Extraction System, IEEE Transactions on Plasma Science, Vol. 45, no. 6, 955-958.

[7] D.A. Fink, T. Kalvas, J. Lettry, Ø. Midttun, D. Noll, (2018) $\mathrm{H}^{-}$extraction systems for CERN's Linac4 $\mathrm{H}^{-}$ion source, Nuclear Inst. and Methods in Physics Research, A 904, 179-187.

[8] Donald W. Mueller, (19-23 October 1981) Proceedings of the Linear Accelerator Conference, Santa Fe, New Mexico, USA, Los Alamos, 281283.
[9] M.M. Abdelrahman, (2012) Factors enhancing production, extraction and focusing of positive ion beams, Ain Shams Engineering Journal 3, 71-78.

[10] Ashraf A. El-Saftawy, Ahmed Elfalaky, Magdi S. Ragheb, Safwat G. Zakhary, (2012) Investigation of beam performance parameters in a pierce-type electron gun, Science and Technology, 2(6): 191197.

[11] Basanta Kumar Das and Anurag Shyam, (2008) Development of compact size penning ion source for compact neutron generator, Rev. Sci. Inst., 79, 123305.

[12] Sae-Hoon Park, Sang-Hun Lee and Yu-Seok Kim, (2018) Simulation Study of the Beam Trajectory Effect on the Magnetic Field Distribution of a Duoplasmatron, Journal of the Korean Physical Society, Vol. 73, No. 8, 1093-1098.

[13] D. A. Dahl, J. E. Delmore and A. D. Appelhans, (1990) SIMION PC/PS2 electrostatic lens design program, Rev. Sci. Inst., 61, 607-609.

[14] R.G., Wilson, and G.R., (1973) Brewer, in the Ion Beam with Applications to Ion Implantation, John Willy and Sons, New York. 